\title{
A sample of X-ray emitting normal galaxies from the BMW-HRI Catalogue ${ }^{\star}$
}

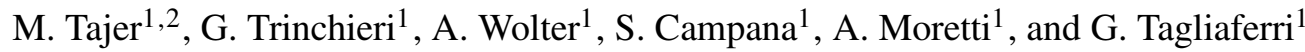 \\ 1 INAF - Osservatorio Astronomico di Brera, via Brera 28, 20121 Milano, Italy \\ e-mail: tajer@brera.mi .astro.it \\ ${ }^{2}$ Università di Milano - Bicocca, Dipartimento di Fisica, P.za della Scienza 3, 20126 Milano, Italy
}

Received 23 June 2004 / Accepted 16 December 2004

\begin{abstract}
We obtained a sample of 143 normal galaxies with X-ray luminosity in the range $10^{38}-10^{43} \mathrm{erg} \mathrm{s}^{-1}$ from the cross-correlation of the ROSAT HRI Brera Multi-scale Wavelet (BMW-HRI) Catalogue with the Lyon-Meudon Extragalactic Database (LEDA). We find that the average X-ray properties of this sample are in good agreement with those of other samples of galaxies in the literature. We selected a complete flux limited serendipitous sample of 32 galaxies from which we derived the $\log N-\log S$ distribution of normal galaxies in the flux range $1.1-110 \times 10^{-14} \mathrm{erg} \mathrm{cm}^{-2} \mathrm{~s}^{-1}$. The resulting distribution is consistent with the Euclidean -1.5 slope. Comparisons with other samples, such as the Extended Medium Sensitivity Survey, the ROSAT All Sky Survey, the XMM-Newton/2dF survey, and the Chandra Deep Field Survey indicate that the $\log N-\log S$ distribution of normal galaxies is consistent with a Euclidean slope over a flux range of about 6 decades.
\end{abstract}

Key words. X-ray: galaxies - galaxies: general - surveys

\section{Introduction}

Detailed X-ray studies of normal galaxies have been possible only with the advent of imaging instruments aboard the Einstein observatory. The Einstein results were summarized in the catalogue and atlas published by Fabbiano et al. (1992), who constructed a large homogeneous sample of 493 galaxies, included in either A Revised Shapley-Ames Catalog of Bright Galaxies (Sandage \& Tammann 1981) or in the Second Revised Catalog of Bright Galaxies (de Vaucouleurs et al. 1976), both targets and objects serendipitously detected in Einstein fields. While representative of the galaxy population (see e.g. Shapley et al. 2001; Eskridge et al. 1995), it was not constructed to be a complete, unbiased sample, so it is likely to contain unknown selection biases.

Elliptical galaxies were found to retain large amounts $\left(10^{8}-10^{11} M_{\odot}\right)$ of hot gas $\left(T \sim 10^{7} \mathrm{~K}\right)$ whose thermal emission dominates their X-ray luminosities, while in normal spirals the integrated contribution of the evolved stellar sources, such as supernova remnants and X-ray binaries, is generally the dominant component (see Fabbiano 1989; Fabbiano et al. 1992; Kim et al. 1992). Extended emission from a hot, gaseous component in spiral galaxies was detected only in some cases (Fabbiano \& Trinchieri 1987; Vogler \& Pietsch 1996; Trinchieri et al. 1988), or associated with starburst activity.

^ Full Table 1, full Fig. 2 and Appendix A are only available in electronic form at http://www. edpsciences.org
Subsequent observations of individual sources made by ROSAT and ASCA confirmed most of the Einstein results and added interesting information on the X-ray properties of normal galaxies in the local universe (see among others Roberts \& Warwick 2000; Read et al. 1997; Brown \& Bregman 1998; Beuing et al. 1999).

With the launch of XMM-Newton and Chandra, the study of the X-ray properties of "normal" galaxies at intermediate $(z \sim 0.1)$ or cosmological distances (Brandt et al. 2001; Hornschemeier et al. 2002, 2003; Georgakakis et al. 2003, 2004a,b; Norman et al. 2004) was made possible, thanks to significantly improved sensitivity and spatial and spectral resolution of the instruments. In spite of the large number of papers, however, a truly complete sample of X-ray emitting normal galaxies in the local universe with a significant number of objects has not been properly discussed in the literature so far. Georgakakis et al. (2003) gave galaxy number density at $F_{0.5-2} \sim 7 \times 10^{-16} \mathrm{erg} \mathrm{cm}^{-2} \mathrm{~s}^{-1}$ from stacking analysis, while Georgakakis et al. (2004a) presented a sample of 26 X-ray sources detected in an area of $\sim 2.5 \mathrm{deg}^{-2}$, of which only 2 were however classified as normal galaxies. Georgakakis et al. (2004b) only recently presented a larger sample of 11 normal galaxies detected in an area of $\sim 4.5 \mathrm{deg}^{-2}$.

Two other samples of "normal" galaxies are available in the literature, selected in the Chandra Deep Fields (Hornschemeier et al. 2003; Norman et al. 2004). However their median redshifts $(z=0.297$, Hornschemeier et al. 2003; and $z=0$ to 
$z=1.3$, Norman et al. 2004) indicates that they should not be considered as "local".

The large database provided by ROSAT has been exploited only marginally to derive unbiased and complete sample of galaxies. Zimmermann et al. (2001) selected a sample of candidate normal galaxies from the ROSAT All Sky Survey (RASS) Bright Source Catalogue (Voges et al. 1999) above a flux limit of about $10^{-12} \mathrm{erg} \mathrm{cm}^{-2} \mathrm{~s}^{-1}(0.1-2.4 \mathrm{keV}$ band). A few samples were derived from the ROSAT Position Sensitive Proportional Counter (PSPC) pointed observations, such as the WGA (White et al. 1994) and the ROSPSPC (ROSAT team 2001), and a new catalogue of galaxies is in progress (G. Peres, private communication). Here we exploited the potential of the Brera Multi-scale Wavelet (BMW-HRI) catalogue (Panzera et al. 2003) to extract a sample of normal galaxies, as we discuss in the next sections. While the PSPC is probably more efficient at detecting faint and extended sources such as galaxies, the sharp core of the HRI point spread function allows us to detect sources in more crowded fields and to measure the extent of bright, small-size sources, providing a good complement to the PSPC data.

\section{The sample}

In order to create a complete, serendipitous sample of galaxies with X-ray emission, we made use of X-ray data from the BMW ROSAT HRI catalogue and optical data from LEDA (Lyon-Meudon Extragalactic Database). The BMW-HRI catalogue consists of 29089 X-ray sources detected in 4303 ROSAT HRI pointed fields with exposure times longer than $100 \mathrm{~s}$ using a multiscale wavelet algorithm (Lazzati et al. 1999; Campana et al. 1999; Panzera et al. 2003). Sources detected with a significance $\geq 4.2 \sigma$ are contained in the catalogue, which provides name, position, count rate, flux, and extension, along with the relative errors. In our study we used the full catalogue, but we excluded X-ray sources in the Trapezium field, which is a rich stellar cluster in the Milky Way where the high density of X-ray sources would prevent proper optical identifications. The BMW-HRI catalogue can be searched via the HEASARC Browse $^{1}$ or via the Brera Observatory web site ${ }^{2}$.

Created in 1983 at Lyon Observatory, $\mathrm{LEDA}^{3}$ was the first database of extragalactic objects and it is continuously updated. It gives a free access to the main astrophysical parameters (coordinates, morphological type, diameter and axis ratio, apparent magnitudes and colors, radial velocity, surface brightness, etc.) for about $10^{6}$ galaxies over the whole sky. The completeness in apparent $B$-magnitude is satisfied up to $m_{B}=15.5$ (see Paturel et al. 1997).

To obtain a representative sample of galaxies we started from the BMW-HRI catalogue and included only serendipitous detections, avoiding the targets. We chose a $3^{\prime}$ radius to

\footnotetext{
${ }^{1}$ http://heasarc.gsfc .nasa.gov/cgi-bin/W3Browse/ w3browse.pl

${ }^{2}$ http://www.merate.mi.astro.it:8081/interroga/ dbServer? $\mathrm{cmd}=\mathrm{bmw} 2$

3 Several mirrors exist to access LEDA; we used the OAB, http://www . brera.mi .astro.it/hypercat/
}



Fig. 1. Displacement between optical and X-ray positions for pointlike sources in the total sample. The solid circle refers to the cross-correlation radius of $20^{\prime \prime}$; the dashed one, with $13^{\prime \prime}$ radius, contains about $90 \%$ of the identifications.

define the typical region of the target, and selected only sources at off-axis angles $\theta>3^{\prime}$. In spite of this location, we had to exclude 12 additional sources that were targets of the observations. We then cross-correlated the positions of the X-ray sources in the BMW-HRI catalogue with those of galaxies present in the LEDA database version of 1999 with a tolerance of $20^{\prime \prime}$, which should be a reasonable guess to detect extended objects like galaxies and to avoid most of spurious coincidences. This criterium is not appropriate for very extended galaxies (e.g. M 31 or M 33), where a large number of sources are detected at distances significantly larger than our tolerance radius. Therefore we could be selecting against large galaxies, if there is no source within a distance of $\sim 20^{\prime \prime}$ from the nucleus. However, this should not be a concern in this study, since the surface density of large galaxies is small; in particular, in the LEDA catalog, the density of galaxies with $D_{25}>3^{\prime}$ is $<3 \times 10^{-2} \mathrm{deg}^{-2}$, which implies about $<10$ in the area we surveyed.

To check the goodness of our choice, we plotted the relative shifts between optical and X-ray positions in Fig. 1. We used only point-like X-ray sources because in extended sources the association with a single optical object could be misleading (e.g. in groups and pairs). About $90 \%$ of the identifications are within $13^{\prime \prime}$ (represented by the dashed circle in Fig. 1).

This is in good agreement with the HRI positional uncertainty, since the best attitude solution guarantees that on average known objects are detected within $10^{\prime \prime}$ of their catalog position, although with possible additional discrepancy mainly in declination (see the ROSAT Handbook at http:// heasarc.gsfc.nasa.gov/docs/rosat/ruh/handbook/ handbook.html). We verified that all X-ray sources with 
$\mathrm{X}$-ray/optical off-set $>10^{\prime \prime}$ are still within the galaxy (i.e. $\mathrm{D}_{25}$ ). We concluded that the association of the X-ray sources with galaxies found by the cross-correlation is sound on positional grounds.

The cross-correlation yielded 399 X-ray sources associated with 281 galaxies. Since constructed field-by-field, the BMWHRI catalogue contains multiple detections of the same source. Therefore we only had 283 distinct X-ray sources associated with galaxies ${ }^{4}$.

To check the results of the cross-correlation, we inspected HRI images for each source and obtained information from the NASA/IPAC Extragalactic Database (NED) to a) eliminate spurious coincidences [15 sources], b) eliminate AGNs [47 objects], and c) select clusters [57 objects].

a. We eliminated a source when the obvious optical counterpart was not a LEDA galaxy, but a background or foreground object. This was checked both using NED and the X-ray/optical contour maps.

b. We excluded objects classified in Véron-Cetty \& Véron (2001) Catalogue as Seyfert 1 galaxies, QSOs, BL Lacs, or AGNs. For Seyfert 2 galaxies, the X-ray emission could have a non nuclear origin, so we eliminated them when we saw from images that the X-ray emission was pointlike and well centered on the nucleus, while we retained those for which we found an extended emission. We also retained starburst galaxies, LINERs and objects that we know from the literature could have a nuclear source but also nonnuclear X-ray emission (e.g. NGC 3079).

c. Several galaxies lie inside a cluster; we inspected X-ray images and eliminated those objects whose X-ray emission was indistinguishable from the cluster's, but we retained galaxies for which emission clearly associated with the galaxy is detected above the cluster background (see PGC 12350 in Fig. 2). We retained galaxies in poor groups even when they are the brightest member since there is still ambiguity in the literature about emission from bright early-type galaxies and groups, which are often analyzed in the same context (see among others Mamon 1992; Dell'Antonio et al. 1994; Pildis et al. 1995; Ponman et al. 1996; Mahdavi et al. 1997; Mulchaey \& Zabludoff 1998; Helsdon \& Ponman 2000; Mulchaey et al. 2003; Osmond \& Ponman 2004; Helsdon \& Ponman 2003; Jones et al. 2003).

Moreover, in 5 cases the same X-ray source was associated with two or more galaxies in a pair or in a group; since we could not discriminate on a positional basis, we chose the brightest galaxy in the pair or in the group.

These selection criteria yielded a total of $195 \mathrm{X}$-ray sources (including multiple detections) associated with 143 galaxies whose properties are listed in Table 1, which is available in electronic form. We show the first page here as an example. Col. (1) gives the BMW-HRI name of the source, Cols. (2) and (3) the position of the X-ray peak, Col. (4) the extension

\footnotetext{
${ }^{4}$ Two distinct sources but of different extent are associated with both NGC 1399 and M 86; we list them in Table 1, but we consider only the largest one for computing fluxes and luminosities.
}

of the X-ray source (or " $p$ " if the source is point-like; asterisks label extended sources for which count rates have been estimated using ad hoc regions), Col. (5) the LEDA galaxy associated to the X-ray source, Col. (6) the name of the galaxy in other common catalogues (e.g. NGC), Col. (7) the morphological type, Col. (8) the distance of galaxy, Col. (9) the apparent $B$-magnitude corrected for galactic extinction, inclination and redshift effects (see Paturel et al. 1997), Col. (10) the HRI count rate with error, Col. (11) the flux with error, Col. (12) the logarithm of X-ray luminosity (count rates and fluxes have been recomputed with respect to values reported in the BMWHRI catalogue; see Sect. 3 for details), and Col. (13) specifies whether the galaxy is in the complete subsample (c), in cluster (Cl) or in group (Gr).

Information about magnitudes and redshifts are from LEDA and NED except for 3 objects that we observed ourselves (see Sect. 5). We calculated distances from redshifts, assuming $H_{0}=50 \mathrm{~km} \mathrm{~s}^{-1} \mathrm{Mpc}^{-1}$; when the heliocentric radial velocity of galaxy was less than $3000 \mathrm{~km} \mathrm{~s}^{-1}$, however, we used distances from Nearby Galaxy Catalogue (Tully 1988) corrected for $H_{0}=50 \mathrm{~km} \mathrm{~s}^{-1} \mathrm{Mpc}^{-1}$.

One hundred and sixteen of the 143 galaxies have redshift and magnitude (including our observations). Nineteen galaxies have $m_{B}$ and no $z, 3$ have $z$ and no $m_{B}$, and 5 have neither $z$ nor $m_{B}$. The redshift distribution ranges from $z=0$ from $z \sim$ 0.15 , but $\sim 90 \%$ of the galaxies have $z<0.07$.

\subsection{The complete serendipitous subsample}

To study the general properties of the sample we needed to derive a subsample with well known completeness criteria and limits. To this end we constructed a complete sample with both $\mathrm{X}$-ray and optical flux limits, and had to consider both the $\mathrm{X}$-ray and the optical completeness criteria. The X-ray completeness is related to the BMW-HRI catalogue that includes all sources with significance $\geq 4.2 \sigma$. To take the optical limits into account, we excluded galaxies fainter than $m_{B}=15.5$, the completeness limit assumed for the LEDA Catalogue (see Paturel et al. 1997).

We also excluded objects at low galactic latitude $\left(|b| \leq 10^{\circ}\right)$ to avoid source confusion in the galactic plane and X-ray sources with off-axis angle $\theta \geq 18^{\prime}$ to match the circular HRI field of view used in the sky coverage computation (see Sect. 7).

The resulting sample of 96 objects is complete both in $\mathrm{X}$-ray and optical at the given limits. However, since galaxies are often in agglomerates, some of them are expected to be related to the targets and therefore not be truly serendipitous. We therefore excluded all sources known to be associated with the target (e.g. galaxies in pairs, groups or clusters; 52 objects in all). When an association was not documented (e.g. from NED, LEDA) we conservatively excluded galaxies at the same redshift as the target (12 objects).

The complete, serendipitous sample of 32 galaxies thus obtained is given in Table 3 and will be used to calculate the $\log N-\log S$ distribution in the local universe $(z<0.07)$, as will be described in detail in Sect. 7 . 

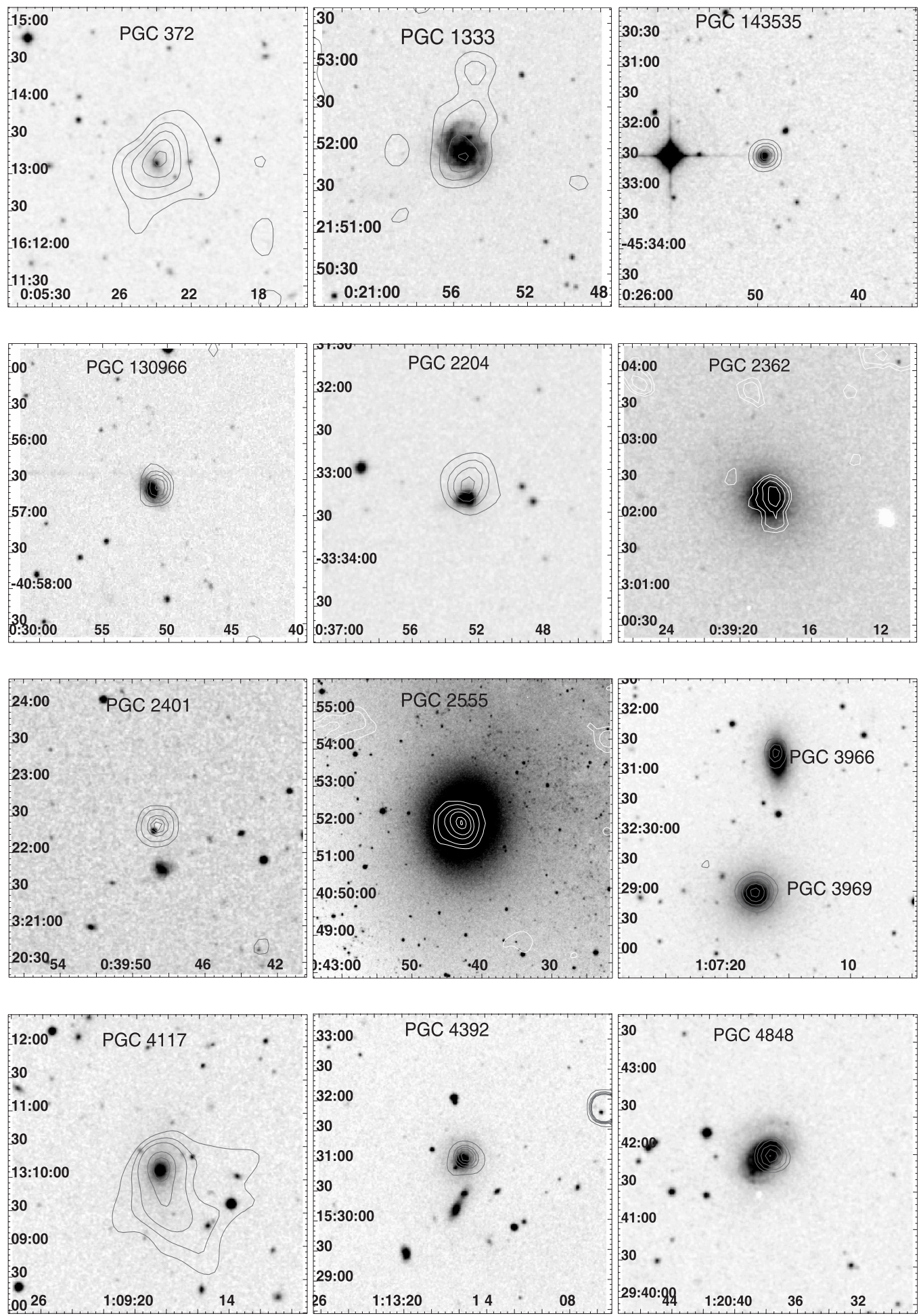

Fig. 2. First page of the figure available in electronic form. It presents the X-ray contours from smoothed X-ray images superimposed onto optical images. Galaxies are generally at the center of the field. Smoothing is done with a Gaussian function of $\sigma=5^{\prime \prime}$ for point-like sources and of $\sigma=10^{\prime \prime}$ for extended sources. 


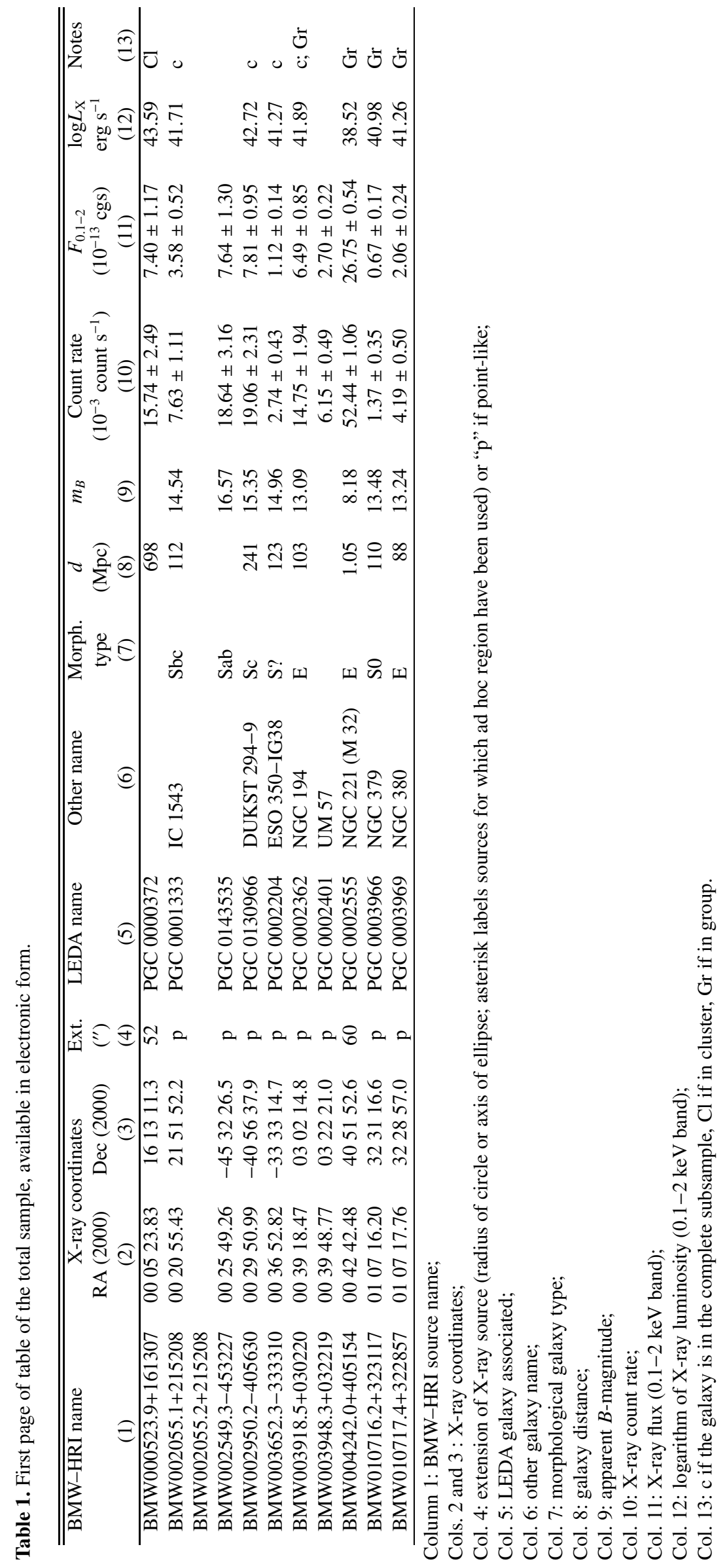




\section{X-ray characterization}

The BMW-HRI catalogue provides count rates derived using the wavelet algorithm in an automated way and under particular assumptions (for details, see Lazzati et al. 1999; Campana et al. 1999). We verified that, while count rates are correctly computed for point-like sources, for very extended sources such as NGC 1399, the extension and count rates given by the algorithm are underestimated. Moreover, when multiple observations are available we could improve the statistics by considering the full set of data. We therefore recalculated all count rates, using the BMW-HRI positions and original HRI data retrieved from the public archives ${ }^{5}$ in pulse height analyzer (PHA) channels 1-10 to increase the signal-to-noise ratio (for a justification of this choice of PHA, see Trinchieri et al. 1997). When multiple observations of the same field are available, we summed the data if they have the same pointing coordinates and comparable exposure times. Otherwise, we typically used the longer exposures or those where the source is closer to the field's center.

\subsection{Count rates}

We classified sources as point-like or extended based on the radial distribution of the emission relative to the shape of the HRI point spread function (PSF; for a description of the ROSAT PSF see Boese 2000) at the corresponding off-axis angle. For the "extended sources" counts were taken from the largest region that contains source counts, in most cases a circle of radius reported in Table 1, evaluated from the radial profile. Given the particular shape of the source, in a few cases we used ellipses to evaluate the counts and give minor and major semiaxes in Table 1. There are also a few extended sources for which ad hoc regions (neither circles nor ellipses) have been used to estimate counts: we label them with an asterisk in Table 1.

For point-like sources the counts were obtained in a circular region centered at the peak of X-ray emission, with radius that includes about $90 \%$ of source counts according to the PSF. The PSF degrades as the angular distance from the center of field increases, so we chose a radius of $r=18^{\prime \prime}$ for $3^{\prime}<\theta \leq 10^{\prime}$, $r=25^{\prime \prime}$ for $10^{\prime}<\theta \leq 15^{\prime}$ and $r=40^{\prime \prime}$ for $\theta>15^{\prime}$, following Boese (2000). We evaluated the background in an annulus concentric to the source radius with radii depending on the off-axis. When the source was particularly faint, we calculated count rates in a circle of radius corresponding to a smaller fraction of the PSF, to increase the signal-to-noise ratio. We then corrected the count rate accordingly following Boese (2000).

We then compared count rates obtained in this way with those reported in the BMW-HRI catalogue and found general agreement, except for some sources whose extension had been largely underestimated by the wavelet algorithm, as stated above. Indeed, our count rates would be equivalent to the "counted count rates" reported in the catalogue, rather than those computed with the wavelet algorithm. In comparison with this quantity, we found a systematically higher count rate

\footnotetext{
5 http://wave.xray.mpe.mpg.de/ and http://heasarc.gsfc.nasa.gov/
}

that is consistent with the larger PHA interval used (1-10 in our analysis and 2-9 in the BMW-HRI catalogue).

The resulting net count rates are given in Table 1, corrected for vignetting and lost counts due to the PSF (for point sources only).

\subsection{Fluxes and luminosities}

The count rates were converted into $0.1-2 \mathrm{keV}$ fluxes using a conversion factor corresponding to a bremsstrahlung spectrum with $k T=5 \mathrm{keV}$, plus the line of sight absorption appropriate for each source from Dickey \& Lockman (1990), which is reported in Table 2. Although this spectrum might not be suitable for all kinds of sources, the flux in the ROSAT energy window depends only weakly on the spectral model assumed, while it is more dependent on low energy absorption. The resulting fluxes are in the range $10^{-14}-10^{-11} \mathrm{erg} \mathrm{cm}^{-2} \mathrm{~s}^{-1}$.

To calculate luminosities, we used distances listed in Table 1. The corresponding range in $L_{\mathrm{X}}$ is $10^{38}-10^{43} \mathrm{erg} \mathrm{s}^{-1}$.

\section{The atlas}

In Fig. 2 we provide an overlay of X-ray contours of the detected galaxies onto optical images from the Digital Sky Survey II (DSS II) available from the ESO archive ${ }^{6}$. This figure is available in electronic edition, we report here the first page as an example. When available, we used optical images obtained with the blue filter, otherwise we used those obtained in the red filter. For PGC 209730 only the DSS I plate is available. Galaxies, ordered in RA and generally at the center of the field, are identified by their PGC name.

$\mathrm{X}$-ray contours are produced from images in the PHA range $1-10$, smoothed using a Gaussian function with $\sigma=5^{\prime \prime}$ for point-like sources and with $\sigma=10^{\prime \prime}$ for extended sources.

\section{Optical observations}

In order to measure redshifts and magnitudes for some of the galaxies of our sample, we made spectroscopical and photometrical observations with the $1.52 \mathrm{~m}$ telescope of the Osservatorio Astronomico di Bologna, at Loiano (Italy) on the nights of the 16th and 17th October 2001. Because of bad atmospheric conditions, we were able to observe only 3 galaxies. A spectrophotometric calibration star was also observed. We present the results obtained in Appendix A, available in electronic edition.

\section{Comparison with literature}

To verify whether the total sample of 143 objects is representative of the X-ray properties of normal galaxies, we calculated $X$-ray luminosities where possible (Table 1 ) and plotted the distributions of $L_{\mathrm{X}}, L_{B}$, the ratio $L_{\mathrm{X}} / L_{B}$ and the $L_{\mathrm{X}}-L_{B}$ relationship for spiral and early-type galaxies. For the 19 galaxies for which the redshift is not known but we have $m_{B}$, the ratio

${ }^{6}$ http://archive.eso.org/dss/dss 
Table 2. Galactic $N_{\mathrm{H}}$ and corresponding unabsorbed flux in the band $0.1-2 \mathrm{keV}$ for $1 \mathrm{count} / \mathrm{s}$, assuming a thermal bremsstrahlung spectral model with $k T=5 \mathrm{keV}$.

\begin{tabular}{lc}
\hline \hline$N_{\mathrm{H}}\left(\mathrm{cm}^{-2}\right)$ & CF $\left(\mathrm{erg} \mathrm{cm}^{-2}\right.$ count $\left.^{-1}\right)$ \\
\hline $1 \times 10^{20}$ & $3.7 \times 10^{-11}$ \\
$2 \times 10^{20}$ & $4.1 \times 10^{-11}$ \\
$5 \times 10^{20}$ & $4.9 \times 10^{-11}$ \\
$8 \times 10^{20}$ & $5.5 \times 10^{-11}$ \\
$3 \times 10^{21}$ & $9.3 \times 10^{-11}$ \\
\hline
\end{tabular}

was calculated from fluxes. We compared our results with those in the literature and found a good general agreement. In particular:

1. The bulk of the galaxies in the sample has an X-ray luminosity between $10^{38} \mathrm{erg} \mathrm{s}^{-1}$ and few $10^{42} \mathrm{erg} \mathrm{s}^{-1}$, in accordance with, e.g., Fabbiano (1989). 7 objects have $L_{\mathrm{X}} \gtrsim 10^{43} \mathrm{erg} \mathrm{s}^{-1}$, but most of them lie in a group, so that the intergalactic medium could contribute to their luminosity; we cannot exclude the presence of an unidentified AGN for some of these objects.

2. For spiral galaxies we found a linear relationship between $\mathrm{X}$-ray and optical luminosities $\left(L_{\mathrm{X}} \propto L_{B}^{1.0 \pm 0.2}\right)$, in agreement with Fabbiano et al. (1992). However, in subsequent, more complete statistical analysis of the 234 "normal" spiral and irregular galaxies reported by Fabbiano et al. (1992), Shapley et al. (2001) and Fabbiano \& Shapley (2002) found that the $L_{X}-L_{B}$ relationship is significantly steeper than linear, with a slope of about 1.5. Our study is based on a much smaller sample (32 spiral and irregular galaxies): as pointed out by Fabbiano \& Shapley (2002), small number statistics could account for the discrepancy.

3. For early-type galaxies we found a steeper relationship than for later types, $L_{\mathrm{X}} \propto L_{B}^{1.6 \pm 0.2}$, consistent with those obtained by Fabbiano et al. (1992) and Eskridge et al. (1995).

4. Values of the X-ray-to-optical ratio in our sample cover roughly the same range $\left(-4<\log \left(L_{X} / L_{B}\right)<0\right.$ with $L_{X}$ and $L_{B}$ in erg s${ }^{-1}$ ) as the spiral galaxies in Shapley et al. (2001) and the early-type galaxies in Eskridge et al. (1995), but their distributions are different. Figure 3 shows the histogram of the X-ray-to-optical ratio of the total sample of 143 galaxies. We will analyze this subject in greater detail in Sect. 7.1.

\section{The $\log N-\log S$ distribution}

The complete serendipitous sample of galaxies derived in Sect. 2.1 (listed in Table 3) was used to calculate the integral flux distribution $(\log N-\log S)$ of normal galaxies with $\mathrm{X}$-ray emission above the X-ray flux limit of the BMW-HRI Catalogue and $B$-magnitude $\leq 15.5$.

The sensitivity of the HRI instrument is not uniform over the entire field of view. Moreover the observing time is different for different fields so we calculated the area surveyed at any given flux (sky coverage).

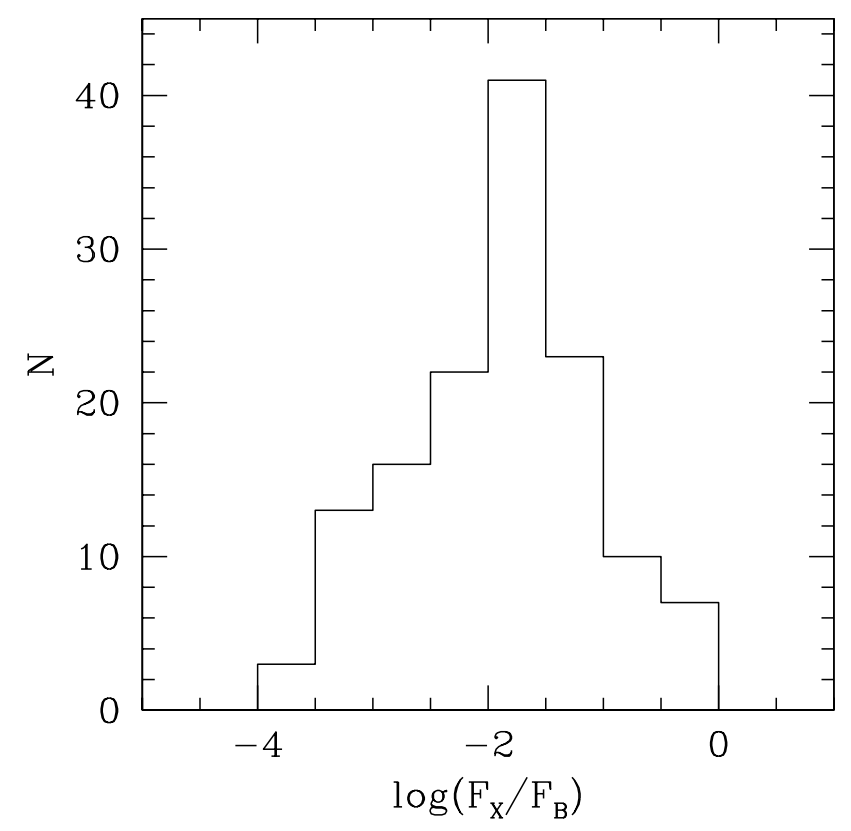

Fig. 3. X-ray-to-optical ratio distribution for the total sample. Both fluxes are in units of $\mathrm{erg} \mathrm{cm}^{-2} \mathrm{~s}^{-1}$.

In the BMW-HRI catalogue the published sky coverage was calculated by means of simulations (see Panzera et al. 2003). In this work we used a sky coverage calculated with the same procedure, but with parameters that reflect our source selection criteria, including therefore only fields with galactic latitude $|b| \geq 10^{\circ}$. We considered only an annular region with $3^{\prime} \leq \theta \leq 18^{\prime}$ : the lower limit accounts for the target region; the upper limit is the largest radius within the field of view of the detector in the assumption of circular symmetry; and we assumed a bremsstrahlung spectrum with $k T=5 \mathrm{keV}$ plus the line of sight absorption.

The resulting sky coverage is plotted in Fig. 4. The maximum area is $\sim 314 \mathrm{deg}^{2}$ and corresponds to fluxes above $\sim 10^{-12} \mathrm{erg} \mathrm{cm}^{-2} \mathrm{~s}^{-1}$. The surveyed area is $\sim 196 \mathrm{deg}^{2}$ at $10^{-13} \mathrm{erg} \mathrm{cm}^{-2} \mathrm{~s}^{-1}$ and $\sim 3 \mathrm{deg}^{2}$ at $\sim 1.1 \times 10^{-14} \mathrm{erg} \mathrm{cm}^{-2} \mathrm{~s}^{-1}$ (the lowest flux for the galaxies in our sample).

For consistency with the sky coverage calculation, fluxes given in Table 3 are derived from the original count rates estimated in the BMW-HRI catalogue, using the wavelet algorithm and PHA channels of HRI from 2 to 9, which are corrected for vignetting and PSF according to Campana et al. (1999) and computed for the energy band $0.5-2 \mathrm{keV}$, the BMW-HRI Catalogue standard.

The integral $\log N-\log S$ distribution of the sample is shown in Fig. 5 and covers two decades in flux, from $\sim 1.1$ to $\sim 110 \times 10^{-14} \mathrm{erg} \mathrm{cm}^{-2} \mathrm{~s}^{-1}$. The overall distribution could be approximated with a slope of $\sim-1.1$ (solid line in Fig. 5). However the Euclidean slope of -1.5 (dashed line) is also consistent with the data: the excess of galaxies at the highest fluxes is small and consistent within the limited statistics. Moreover, at the lower fluxes, there could be some problems with incompleteness, as we discuss below. 
Table 3. Galactic column density (from Dickey \& Lockman 1990), BMW count rates and fluxes for galaxies in the complete serendipitous sample, ordered by increasing flux.

\begin{tabular}{|c|c|c|c|}
\hline LEDA name & $\begin{array}{c}N_{\mathrm{H}} \\
\left(10^{20} \mathrm{~cm}^{-2}\right)\end{array}$ & $\begin{array}{r}\text { Count rate } \\
\left(10^{-3} \text { count s }^{-1}\right)\end{array}$ & $\begin{array}{c}F_{0.5-2} \\
\left(10^{-13} \mathrm{erg} \mathrm{cm}^{-2} \mathrm{~s}^{-1}\right)\end{array}$ \\
\hline 0157406 & 4.9 & $0.37 \pm 0.07$ & $0.12 \pm 0.02$ \\
\hline 0068536 & 2.5 & $1.11 \pm 0.34$ & $0.32 \pm 0.09$ \\
\hline 0025825 & 4.2 & $1.12 \pm 0.22$ & $0.35 \pm 0.06$ \\
\hline 0042833 & 2.0 & $1.31 \pm 0.23$ & $0.35 \pm 0.05$ \\
\hline 0028990 & 0.8 & $1.50 \pm 0.30$ & $0.36 \pm 0.07$ \\
\hline 0005323 & 3.8 & $1.33 \pm 0.28$ & $0.41 \pm 0.09$ \\
\hline 0010446 & 3.1 & $1.48 \pm 0.24$ & $0.43 \pm 0.06$ \\
\hline 0057078 & 2.3 & $1.63 \pm 0.28$ & $0.44 \pm 0.08$ \\
\hline 0069338 & 8.8 & $1.24 \pm 0.20$ & $0.46 \pm 0.07$ \\
\hline 0038773 & 2.0 & $1.70 \pm 0.11$ & $0.46 \pm 0.03$ \\
\hline 0001333 & 4.2 & $1.55 \pm 0.27$ & $0.48 \pm 0.09$ \\
\hline 0007289 & 5.5 & $1.71 \pm 0.26$ & $0.56 \pm 0.10$ \\
\hline 0070861 & 2.6 & $2.11 \pm 0.38$ & $0.61 \pm 0.12$ \\
\hline 0018991 & 5.4 & $2.18 \pm 0.32$ & $0.70 \pm 0.10$ \\
\hline 0004117 & 4.0 & $2.36 \pm 0.23$ & $0.73 \pm 0.06$ \\
\hline 0043675 & 3.9 & $2.49 \pm 0.37$ & $0.77 \pm 0.12$ \\
\hline 0002204 & 1.9 & $3.23 \pm 0.23$ & $0.87 \pm 0.05$ \\
\hline 0045318 & 1.0 & $3.78 \pm 0.60$ & $0.91 \pm 0.14$ \\
\hline 0046432 & 3.2 & $3.13 \pm 0.66$ & $0.91 \pm 0.20$ \\
\hline 0047432 & 2.3 & $3.61 \pm 0.64$ & $0.97 \pm 0.16$ \\
\hline 0130966 & 2.4 & $4.41 \pm 0.81$ & $1.19 \pm 0.22$ \\
\hline 0016574 & 5.9 & $3.73 \pm 0.51$ & $1.23 \pm 0.17$ \\
\hline 0005324 & 3.8 & $4.34 \pm 0.51$ & $1.35 \pm 0.16$ \\
\hline 0057728 & 1.5 & $5.11 \pm 0.39$ & $1.38 \pm 0.11$ \\
\hline 0013368 & 1.4 & $7.22 \pm 1.41$ & $1.73 \pm 0.24$ \\
\hline 0029050 & 0.8 & $10.20 \pm 0.70$ & $2.45 \pm 0.17$ \\
\hline 0002362 & 2.7 & $9.06 \pm 0.99$ & $2.63 \pm 0.29$ \\
\hline 0004848 & 6.1 & $9.26 \pm 2.43$ & $3.06 \pm 0.66$ \\
\hline 0063122 & 7.9 & $11.02 \pm 1.42$ & $3.97 \pm 0.36$ \\
\hline 0006367 & 3.0 & $14.80 \pm 0.60$ & $4.29 \pm 0.17$ \\
\hline 0017451 & 7.5 & $15.66 \pm 1.72$ & $5.64 \pm 0.72$ \\
\hline 0028995 & 4.8 & $34.20 \pm 2.00$ & $10.94 \pm 0.64$ \\
\hline
\end{tabular}

\subsection{Comparison with the literature: $X$-ray-to-optical ratio distribution}

Before comparing our $\log N-\log S$ distribution with those of other samples in the literature, we need to investigate the X-ray-to-optical ratio distribution of our complete sample more throughly. We consider samples derived from ROSAT and Einstein observations that cover a flux range similar to ours; samples derived from XMM-Newton and Chandra surveys, which cover a flux range significantly fainter than ours, will be considered later, in the discussion of the $\log N-\log S$ (Sect. 7.2).

The best available comparison could be with the sample of candidate normal galaxies found by Zimmermann et al. (2001) in the ROSAT All Sky Survey (RASS) and with the normal galaxies found in the Einstein Extended Medium Sensitivity Survey (EMSS; Gioia et al. 1990), both X-ray selected. An effective optically selected sample for comparison is the Einstein galaxy sample (Fabbiano et al. 1992; Shapley et al. 2001; Eskridge et al. 1995).

Zimmermann et al. (2001) made a correlation study of the RASS Bright Source Catalogue (Voges et al. 1999) with the
Catalogue of Principal Galaxies (Paturel et al. 1989), from which they selected a sample of 198 candidate galaxies, i.e. $\mathrm{X}$-ray sources whose optical counterpart was not designated as AGN in the literature. These selection criteria are similar to ours, and the Catalogue of Principal Galaxies is a preliminary version of the current LEDA database, so the two samples can be easily compared; however most of their sources have fluxes above $10^{-12} \mathrm{erg} \mathrm{cm}^{-2} \mathrm{~s}^{-1}$ (computed in the $0.1-2.4 \mathrm{keV}$ band, assuming a power law spectrum with photon index $\Gamma=2.3$ ).

The EMSS was obtained from analysis of 1453 images of the imaging proportional counter (IPC) on board the Einstein Observatory. The survey covers an area of $778 \mathrm{deg}^{2}$ at $|b|>$ $20^{\circ}$ with limiting sensitivity ranging from $\sim 5 \times 10^{-14}$ to $\sim 3 \times 10^{-12} \mathrm{erg} \mathrm{cm}^{-2} \mathrm{~s}^{-1}(0.3-3.5 \mathrm{keV}$ band). 835 serendipitous sources were detected at or above the $4 \sigma$ level (see Gioia et al. 1990; Stocke et al. 1991). Among these, 17 were identified as normal galaxies.

The Einstein sample is the catalogue of normal galaxies observed by the Einstein satellite, compiled by Fabbiano et al. (1992) and reanalyzed by Eskridge et al. (1995, for early-type galaxies) and by Shapley et al. (2001, for spiral galaxies); we no longer distinguish the two samples here since early and late 


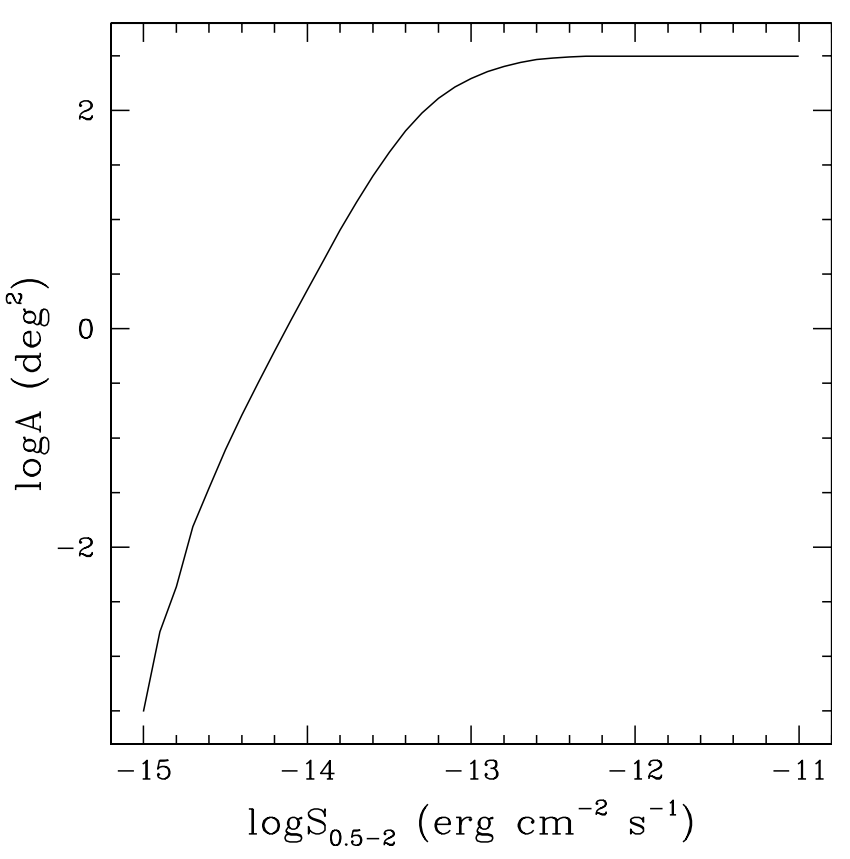

Fig. 4. Sky coverage for the fields at galactic latitude $|b| \geq 10^{\circ}$ and off-axis angle $3^{\prime} \leq \theta \leq 18^{\prime}$, computed by assuming a thermal bremsstrahlung spectrum with $k T=5 \mathrm{keV}$ and galactic line of sight absorption.

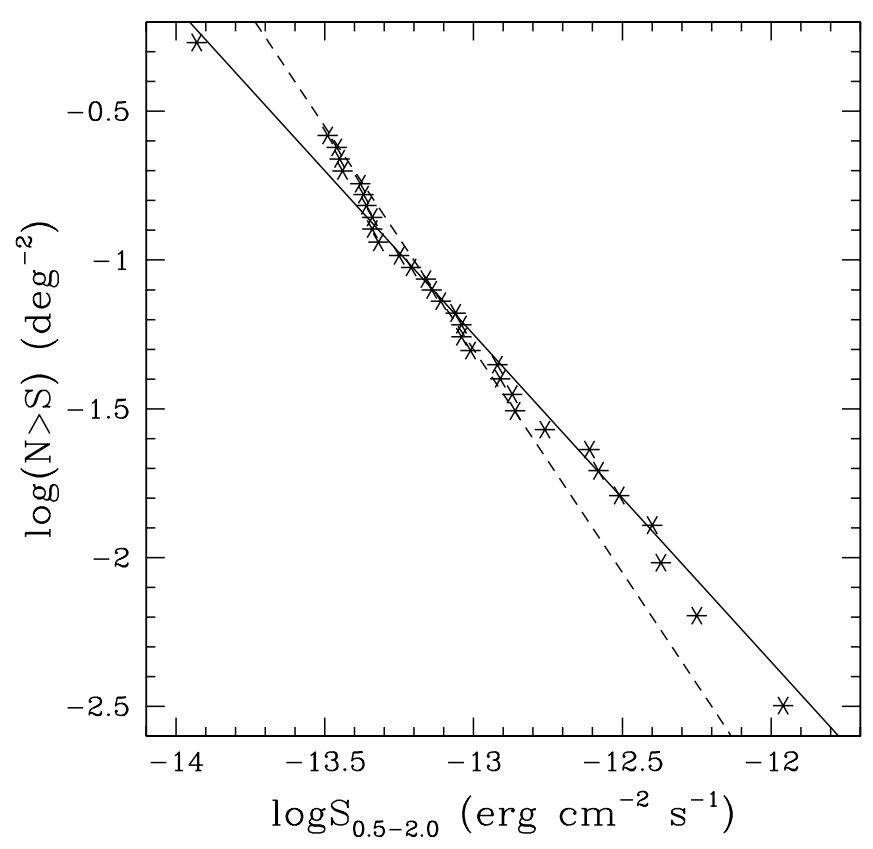

Fig. 5. The integral $\log N-\log S$ distribution for the complete serendipitous subsample (asterisks). The solid line represents the -1.1 slope and the dashed line the Euclidean -1.5 slope.

type galaxies are mixed in the BMW-HRI sample. We consider here only detections in the Einstein sample; the exclusion of upper limits should not affect the results, since their distribution is consistent with that of detections (see Fig. 5 in Shapley et al. 2001).

In Fig. 6 we plot the distribution of $F_{\mathrm{X}}$ and $F_{B}$ values from all samples considered. For consistency with the values

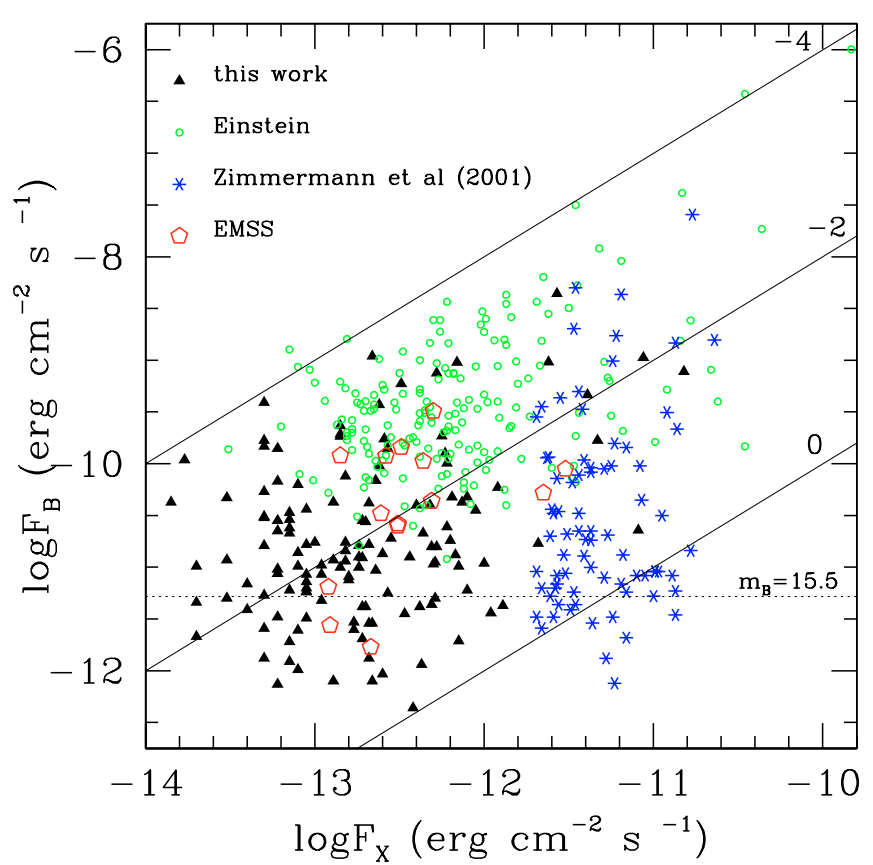

Fig. 6. B flux versus X-ray flux for our total sample (solid triangles), for the Einstein sample (empty circles), for the Zimmermann et al. (2001) sample galaxies (asterisks), and for the EMSS galaxies (empty pentagons). Solid lines correspond to $\log \left(F_{\mathrm{X}} / F_{B}\right)=0,-2,-4$, as indicated; the horizontal dashed line is the $m_{B}=15.5$ limit. Only detections in the Einstein sample and only objects with $F_{0.1-2}>2 \times$ $10^{-12} \mathrm{erg} \mathrm{cm}^{-2} \mathrm{~s}^{-1}$ and $\log L_{\mathrm{X}}<42.7$ in the Zimmermann et al. (2001) sample are plotted (see text).

in Table 1, all fluxes are converted to the $0.1-2 \mathrm{keV}$ energy band, using a thermal bremsstrahlung spectrum with temperature $k T=5 \mathrm{keV}$ and $N_{\mathrm{H}}=3 \times 10^{20} \mathrm{~cm}^{-2}$.

Figure 6 clearly indicates that galaxies belonging to different samples populate different regions in the plot. Our sample (solid triangles) has an X-ray flux range between $10^{-14}$ and $10^{-11} \mathrm{erg} \mathrm{cm}^{-2} \mathrm{~s}^{-1}$ and $\log \left(F_{\mathrm{X}} / F_{B}\right)$ between -4 and 0 (see also Fig. 3). The bulk of Einstein galaxies (empty circles) is typically at higher average fluxes and at lower values of $\log \left(F_{\mathrm{X}} / F_{B}\right)$ (between -4 and -2$)$ compared to our distribution and to the X-ray selected samples in general. This sample is the largest; it is effectively optically selected and reasonably clean of contamination from AGN (Shapley et al. 2001). However, since it is not complete, it might not provide true distribution of the X-ray-to-optical ratios.

The second largest sample is derived from Zimmermann et al. (2001), with an additional flux limit $F_{X}>2 \times$ $10^{-12} \mathrm{erg} \mathrm{cm}^{-2} \mathrm{~s}^{-1}$ (for completeness, see discussion below) and an upper limit in luminosity at $\log L_{\mathrm{X}}=42.7 \mathrm{erg} \mathrm{s}^{-1}$, the highest luminosity in our complete sample, comparable to their limit to exclude potential AGNs from the sample. The distribution of candidate galaxies in Zimmermann et al. (2001) (asterisks) is significantly different from that of the Einstein sample and extends at $\log \left(F_{\mathrm{X}} / F_{B}\right)>0$. The $F_{\mathrm{X}} / F_{B}$ distribution for EMSS galaxies (empty pentagons) is at intermediate values and more consistent with that of our sample.

Also plotted in Fig. 6 is the optical flux limit applied to our sample, $m_{B}=15.5$. It is evident that excluding galaxies 
fainter than this for the $\log N-\log S$ calculation has an effect that increases as flux decreases. We attepted to quantify it in order to correct the curve for lost objects. Unfortunately we could not properly estimate the correction because none of the galaxy samples available allows us to derive the true distribution of X-ray-to-optical ratios of normal galaxies. The EMSS should represent the true $F_{\mathrm{X}} / F_{B}$ distribution, but given the small number of galaxies (17, of which only 15 have $B$-magnitude), statistical errors are large. The difference in distribution of ratios between the two larger samples in Fig. 6 suggests that they might be affected by opposite biases; the Zimmermann et al. (2001) sample is likely to contain unclassified AGNs, while the Einstein sample could lose objects at the highest X-ray-to-optical ratios. If we use the three samples to estimate how many galaxies are lost as a function of $\mathrm{X}$ ray flux, we find that corrections to the $\log N-\log S$ are small and the recomputed curve is consistent with the Euclidean slope in the observed flux range.

We also tried to estimate the correction by considering optically fainter galaxies. At $m_{B}=16$, LEDA is about $90 \%$ complete (see Fig. 7 in Paturel et al. 1997). If we include galaxies down to this flux limit, we only add 4 objects to our serendipitous sample, distributed over the whole range of X-ray fluxes; therefore, their inclusion influences only slightly the normalization, not the slope of the distribution.

We conclude that since the effects introduced by the optical limit are small, the $\log N-\log S$ we derive is consistent with the Euclidean slope.

\subsection{Comparison with the literature: $\log N-\log S$}

Figure 7 shows the comparison between the $\log N-\log S$ derived above with several available from the literature. All fluxes are recomputed in the $0.5-2 \mathrm{keV}$ range that we use. We find excellent agreement with other samples that cover similar or brighter flux ranges than the present sample.

The candidate galaxies (Zimmermann et al. 2001) appear to connect smoothly with the Euclidean extrapolation of the BMW-HRI $\log N-\log S$ above $10^{-12} \mathrm{erg} \mathrm{cm}^{-2} \mathrm{~s}^{-1}$. We interpret the flattening observed in the Zimmermann et al. (2001) data at lower fluxes as a result of their selection criteria. In fact, they indicate a $\geq 90 \%$ completeness for count rates $\geq 0.1$ count s$^{-1}$, which converts to a flux of $F_{0.5-2} \sim$ $7 \times 10^{-13} \mathrm{erg} \mathrm{cm}^{-2} \mathrm{~s}^{-1}$.

Although small, the EMSS is a truly complete sample, since it is serendipitously X-ray selected and $\sim 96 \%$ identified (Gioia et al. 1990; Stocke et al. 1991; Maccacaro et al. 1994). The EMSS appears to be Euclidean and almost coincident with our curve for fluxes above $10^{-13} \mathrm{erg} \mathrm{cm}^{-2} \mathrm{~s}^{-1}$.

We also extended the comparison to include samples at fainter fluxes. Georgakakis et al. (2004a) compute the $\log N-$ $\log S$ of sources in the XMM-Newton/2dF survey, obtained with the EPIC instrument on board the XMM-Newton satellite. This survey covers an area of about $2.5 \mathrm{deg}^{2}$ to the flux limit $\sim 10^{-14} \mathrm{erg} \mathrm{cm}^{-2} \mathrm{~s}^{-1}$ in the $0.5-8 \mathrm{keV}$ band (or $F_{0.5-2} \sim$ $\left.5 \times 10^{-15} \mathrm{erg} \mathrm{cm}^{-2} \mathrm{~s}^{-1}\right)$. They find two "normal" galaxies in

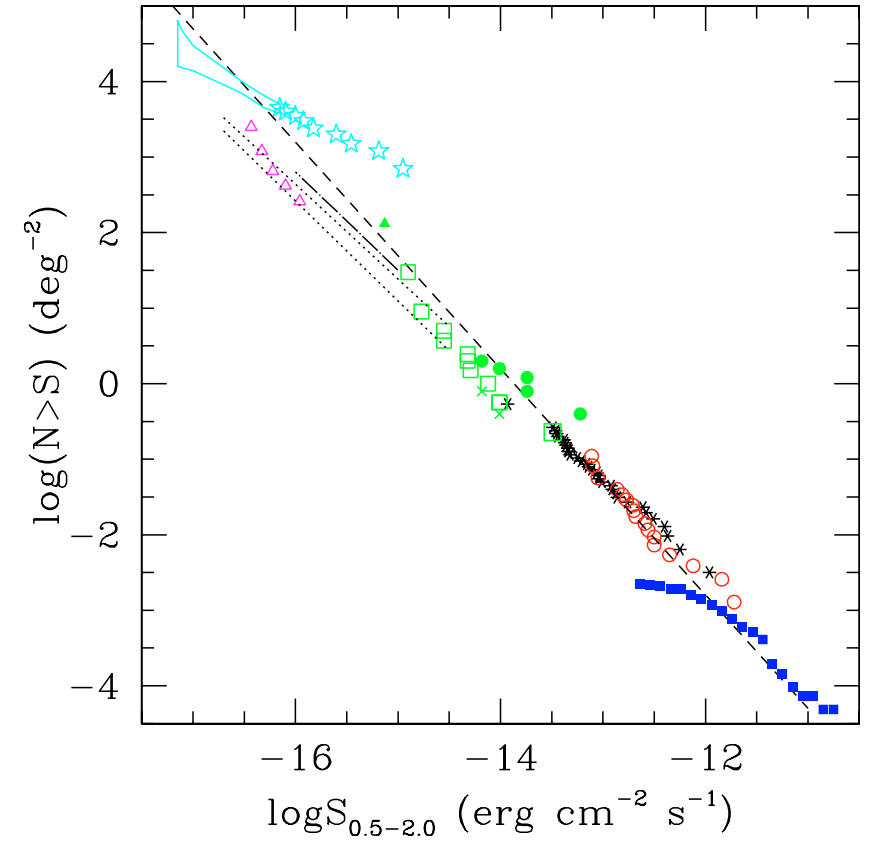

Fig. 7. Comparison of the $\log N-\log S$ distribution obtained from the present sample (asterisks) with several other relations for normal galaxies from the literature. The dashed line represents the Euclidean relationship of Fig. 5 extrapolated to the full flux range considered. Symbols refer to: EMSS galaxies (empty circles); candidate galaxies in Zimmermann et al. (2001; solid squares); normal galaxies (crosses), including LLAGN (solid circles, Georgakakis et al. 2004a), and stacking analysis (solid triangles, Georgakakis et al. 2003) found in the $X M M-N e w t o n / 2 \mathrm{dF}$ survey; XMM-Newton/Sloan DSS normal galaxies from Georgakakis et al. (2004b; empty squares); Chandra Deep Fields results from Hornschemeier et al. (2003; empty triangles); optimistic or pessimistic curves given by Bauer et al. (2004) (upper/lower dotted line); Bayesian sample of Norman et al. (2004) reported by Ranalli et al. (2004; dot - dashed line). Open stars and horned-shaped box indicate the detections and fluctuation analysis predictions of Miyaji \& Griffiths (2002).

their sample, which implies a density of $<1$ source at $F_{\mathrm{X}} \sim$ $10^{-14} \mathrm{erg} \mathrm{cm}^{-2} \mathrm{~s}^{-1}$, lower than what we find.

However, the sample of Georgakakis et al. (2004a) contains three additional galaxies (at $z \leq 0.1$ with $L_{0.5-8} \sim 10^{42} \mathrm{erg} \mathrm{s}^{-1}$ ) that the authors do not consider because they might contain Low Luminosity AGNs (LLAGNs, see their Fig. 2). Since we cannot exclude that our sample also contains a few LLAGNs (see above and Sect. 6), we should consider these objects for better comparison with our sample. With inclusion of these objects the $\log N-\log S$ matches ours better.

Also shown in Fig. 7 are the constraints from the stacking analysis results of Georgakakis et al. (2003) at fainter fluxes, computed from optically selected galaxies at a mean redshift of $z \sim 0.1$. The point derived from the total sample considered is in excellent agreement with our $\log N-\log S$.

Recently Georgakakis et al. (2004b) have presented a pilot sample of normal galaxies serendipitously detected in XMMNewton public observations. They find 11 "normal" galaxy candidates with luminosities below $10^{42} \mathrm{erg} \mathrm{s}^{-1}$ over an area of $\sim 4.5 \mathrm{deg}^{2}$. They find that the $\log N-\log S$ derived from this 
sample (plotted in Fig. 7) is again almost Euclidean in slope, although at a smaller normalization than ours.

We also plot the results from deeper surveys, using data from Hornschemeier et al. (2003), Norman et al. (2004) as reported by Ranalli et al. (2004), and Bauer et al. (2004) derived from the Chandra Deep Fields. All these relations fall close to, though generally below, the extrapolation from our sample. Different authors derive different slopes for their samples, but they are all consistent with the Euclidean one.

The lower normalizations found in these latter samples could be explained in part by the combined effects of a) more stringent criteria to minimize contamination from the AGNs, even though of low luminosity, and b) different relative occurrence of the galaxy types (spiral/starburst vs early types).

As already discussed, some residual contamination from low luminosity AGNs could be present in the sample we considered, since we have little information on the optical spectra and could only reject known AGNs. We note, however, that Zimmermann et al. (2001) apply the same criterium adopted by Georgakakis et al. (2004a), namely an X-ray to optical luminosity ratio smaller than $10^{-2}$, and that the EMSS sample, which is well studied optically, should not be contaminated by AGNs. We nevertheless considered discarding sources in our sample that have a $\log \left(F_{0.5-2} / F_{B}\right)<-2$. We have 7 objects that violate this limit, mostly at the high flux end. The resulting $\log N-\log S$ relation is slightly steeper, but consistent with that presented in Fig. 5, and would not significantly lower the normalization of the "Euclidean" curve plotted. However the location of the points from Georgakakis et al. (2004a) that consider/discard possible contamination from LLAGNs gives an idea of the possible uncertainties involved.

The effect of different relative contributions from the early/late types is more complicated to assess. The lowest flux points (Hornschemeier et al. 2003) are derived from late type galaxies, so they could underrepresent the total population. However, Bauer et al. (2004) suggest that the early type galaxies follow a flatter distribution; in any case their $\log N-\log S$ is always below those from starburst/quiescent galaxies (see their Fig. 9), so their contribution could in fact be negligeable at the fainter fluxes. In the range covered by Georgakakis et al. (2004b), there is only one early type galaxy (but the sample is very small), while our sample has a sizeable fraction of early types (1/3 among the objects with a morphological classification); and the percentage increases to $\sim 50 \%$ in the "candidate normal galaxies" of Zimmermann et al. (2001), for which there is moreover no apparent significant different in the two slopes. The stacking analysis results from Georgakakis et al. (2003), derived separately for $\mathrm{E} / \mathrm{S} 0$ and $\mathrm{Sa}-\mathrm{Scd}$, bracket the extrapolation of the Euclidean $\log N-\log S$ obtained from brighter samples, with Sa-Scd in better agreement with the samples at low fluxes. Since the emission from early and late type galaxies is due to significantly different processes (Fabbiano 1989; Fabbiano et al. 1992; Kim et al. 1992; Eskridge et al. 1995; Shapley et al. 2001), a different evolution is not out of the question. Assessment of the $\operatorname{local} \log N-\log S$ separately for each morphological type would be a step toward better understanding of the properties of galaxies as a class, and would provide stronger constraints for the investigation of normal galaxies at higher redshifts, which is beyond the scope of the present work. For now, we simply notice how remarkable it is that, in spite of the different selection criteria and instruments used to define all the samples considered, the surface density of normal galaxies is consistent with a single Euclidean distribution for about 6 decades in flux (from $\sim 10^{-11}$ to $\sim 3 \times 10^{-17} \mathrm{erg} \mathrm{cm}^{-2} \mathrm{~s}^{-1}$ ).

\section{Conclusions}

We present the results for an "almost serendipitous" sample of 143 X-ray emitting normal galaxies selected from the cross-correlation of the BMW-HRI Catalogue and the LEDA database. Isointensity X-ray contours are overlayed onto the optical images for all galaxies and presented in an atlas in Fig. 2. The X-ray characteristics of the sample, listed in Table 1, are derived uniformly way and are used in comparison with other samples in the literature. We find that the general properties of the total sample are in good agreement with those already known for normal galaxies.

We also present a complete subsample of 32 truly serendipitous sources in the local universe $(z<0.07)$, for which we derive the $\log N-\log S$ distribution in the flux range between $\sim 1.1$ and $110 \times 10^{-14} \mathrm{erg} \mathrm{cm}^{-2} \mathrm{~s}^{-1}$ in the $0.5-2 \mathrm{keV}$ energy band. We find that this relation is consistent with the Euclidean distribution.

Moreover, we find good agreement between our $\log N-$ $\log S$ and those derived from ROSAT PSPC and Einstein data at similar or brighter fluxes and from XMM-Newton and Chandra at fainter fluxes; the overall distribution appears to be consistent with a Euclidean slope for about 6 decades in flux, from $\sim 3 \times 10^{-17}$ to $10^{-11} \mathrm{erg} \mathrm{cm}^{-2} \mathrm{~s}^{-1}$. The normalizations of different samples are consistent within a factor of $\sim 2$.

Although with limited statistics, this work provides a first estimate of the number density of sources identified with normal galaxies in the nearby universe, in a flux range (both optical and X-ray) easily accessible for detailed follow up observations. This will allow us to provide a solid basis for studying and classifying objects found in deeper surveys.

While current efforts are mainly focused on probing the distant universe to determine the relevance of normal galaxies as a class at very faint fluxes, the success of these studies also depends on the constraints given by the bright flux end of the number counts, which has still not been studied well. This is particularly relevant at the lowest fluxes where number counts approach total source density (e.g. detections and fluctuation analysis results of Miyaji \& Griffiths 2002, see Hornschemeier et al. 2002, and Fig. 7). The sample derived here will therefore be instrumental in studies of both the cosmological evolution of galaxies and the contribution of this class of sources to the $\mathrm{X}$-ray background.

Acknowledgements. We thank Dr. R. Panzera for her invaluable help, in particular in the extraction of our sample from the BMW-HRI catalogue, and Dr. U. Zimmermann for useful discussion on their work and for providing us with the original data for their $\log N-\log S$ that we use in Fig. 7.

We acknowledge partial financial support from the Italian Space Agency (ASI).

This research made use of the Lyon-Meudon Extragalactic Database 
(LEDA), used the NASA/IPAC Extragalactic Database (NED) which is operated by the Jet Propulsion Laboratory, California Institute of Technology, under contract with the National Aeronautics and Space Administration, and the SAOimage ds9 developed by the Smithsonian Astrophysical Observatory.

The compressed files of the "Palomar Observatory - Space Telescope Science Institute Digital Sky Survey" of the northern sky, based on scans of the Second Palomar Sky Survey are copyright (c) 1993-2000 by the California Institute of Technology and are distributed by agreement. All Rights Reserved.

\section{References}

Bauer, F. E., Alexander, D. M., Brandt, W. N., et al. 2004, accepted for publication in AJ [arXiv: astro-ph/0408001]

Beuing, J., Döbereiner, S., Böhringer, H., \& Bender, R. 1999 , MNRAS, 302, 209

Boese, F. G. 2000, A\&AS, 141, 507

Brandt, W. N., Hornschemeier, A. E., Alexander, D. M., et al. 2001, AJ, 122, 1

Brown, B. A., \& Bregman, J. N. 1998, ApJ, 495, L75

Campana, S., Lazzati, D., Panzera, M. R., \& Tagliaferri, G. 1999, ApJ, 524, 423

David, L. P., Jones, C., \& Forman, W. 1992, ApJ, 388, 82

Dell'Antonio, I. P., Geller, M. J., \& Fabricant, D. G. 1994, AJ, 107 427

Dickey, J. M., \& Lockman, F. J. 1990, ARA\&A, 28, 215

Eskridge, P. B., Fabbiano, G., \& Kim, D.-W. 1995, ApJS, 97, 141

Fabbiano, G. 1989, ARA\&A, 27, 87

Fabbiano, G., \& Shapley, A. 2002, ApJ, 565, 908

Fabbiano, G., \& Trinchieri, G. 1987, ApJ, 315, 46

Fabbiano, G., Kim, D.-W., \& Trinchieri, G. 1992, ApJS, 80, 531

Georgakakis, A., Georgantopoulos, I., Stewart, G. C., Shanks, T., \& Boyle, B. J. 2003, MNRAS, 344, 161

Georgakakis, A., Georgantopoulos, I., Vallbe, M., et al. 2004a, MNRAS, 349, 135

Georgakakis, A., Georgantopoulos, I., Basilakos, S., Plionis, M., \& Kolokotronis, V. 2004b, MNRAS, in press [arXiv:astro-ph/0407387]

Gioia, I. M., Maccacaro, T., Schild, R. E., et al. 1990, ApJS, 72, 567

Helsdon, S. F., \& Ponman, T. J. 2000, MNRAS, 319, 933

Helsdon, S. F., \& Ponman, T. J. 2003, MNRAS, 340, 485

Hornschemeier, A. E., Brandt, W. N., Alexander, D. M., et al. 2002, ApJ, 568, 82

Hornschemeier, A. E., Bauer, F. E., Alexander, D. M., et al. 2003, AJ, 126,575

Jones, L. R., Ponman, T. J., Horton, A., et al. 2003, MNRAS, 343, 627

Kim, D.-W., Fabbiano, G., \& Trinchieri, G. 1992, ApJS, 80, 645
Lazzati, D., Campana, S., Rosati, P., Panzera, M. R., \& Tagliaferri, G. 1999, ApJ, 524, 414

Maccacaro, T., Gioia, I. M., Wolter, A., Zamorani, G., \& Stocke, J. T. 1988, ApJ, 326, 68

Maccacaro, T., Wolter, A., McLean, B., et al. 1994, ApL\&C, 29, 267

Mahdavi, A., Böhringer, H., Geller, M. J., \& Ramella, M. 1997, ApJ, 483,68

Mamon, G. A. 1992, in Physics of Nearby Galaxies: Nature or Nurture?, ed. T. X. Thuan, C. Balkowski, \& J. Tran Thanh Van (Gif-sur-Yvette: Éditions Frontières), 367

Miyaji, T., \& Griffiths, R. E. 2002, ApJ, 564, L5

Mulchaey, J. S., \& Zabludoff, A. I. 1998, ApJ, 496, 73

Mulchaey, J. S., Davis, D. S., Mushotzky, R. F., \& Burstein, D. 2003, ApJS, 145, 39

Norman, C., Ptak, A., Hornschemeier, A., et al. 2004, ApJ, 607, 721

Osmond, J. P. F., \& Ponman, T. J. 2004, MNRAS, 350, 1511

Panzera, M. R., Campana, S., Covino, S., et al. 2003, A\&A, 399, 351

Paturel, G., Andernach, H., Bottinelli, L., et al. 1997, A\&AS, 124, 109

Paturel, G., Fouqué, P., Bottinelli, L., \& Gouguenheim, L. 1989, A\&AS, 80299

Pildis, R. A., Bregman, J. N., \& Evrard, A. E. 1995, ApJ, 443, 514

Ponman, T. J., Bourner, P. D. J., Ebeling, H., \& Böhringer, H. 1996, MNRAS, 283, 690

Ranalli, P., Comastri, A., \& Setti, G. 2004, Proc. Conf. Multiwavelength AGN Surveys, Cozumel, Mexico 2003 [arXiv:astro-ph/0404087]

Read, A. M., Ponman, T. J., \& Strickland, D. K. 1997, MNRAS, 286, 626

Roberts, T. P., \& Warwick, R. S. 2000, MNRAS, 315, 98

Sandage, A., \& Tammann, G. A. 1981, Revised Shapley-Ames Catalog of Bright Galaxies, Carnegie Inst. of Washington, Publ. 635

Shapley, A., Fabbiano, G., \& Eskridge, P. B. 2001, ApJS, 137, 139

Stocke, J. T., Morris, S. L., Gioia, I. M., et al. 1991, ApJS, 76, 813

Trinchieri, G., Noris, L., \& di Serego Alighieri, S. 1997, A\&A, 326, 565

Trinchieri, G., Fabbiano, G., \& Peres, G. 1988, ApJ, 325, 531

Tully, R. B. 1988, Nearby galaxies catalog (Cambridge and New York: Cambridge University Press)

de Vaucouleurs, G., de Vaucouleurs, A., \& Corwin, J. R. 1976, Second reference catalogue of bright galaxies (Austin: University of Texas Press)

Véron-Cetty, M.-P., \& Véron, P. 2001, A\&A, 374, 92

Voges, W., Aschenbach, B., Boller, T., et al. 1999, A\&A, 349, 389

Vogler, A., \& Pietsch, W. 1996, A\&A, 311, 35

White, N. E., Giommi, P., \& Angelini, L. 1994, Bull. Amer. Astron. Soc., 26, 1372

Zimmermann, H.-U., Boller, T., Döbereiner, S., \& Pietsch, W. 2001, A\&A, 378, 30 